\section{Cff}

a conformational

change in the

intracellular

portion of CD3

might constitute

part of the

CD3-mediated

activation signal.

\title{
CD3 conformation is crucial for signalling
}

$\mathrm{T}$ cell activation requires a $\mathrm{T}$ cell receptor (TCR) to recognize its cognate peptide in the context of an MHC molecule. In addition, the association of CD3 with the TCRpeptide-MHC complex transmits the activation signal to intracellular signalling molecules to initiate a signalling cascade in the T cell. The mechanism by which CD3 signals that the TCR has recognized antigen is not known, but two hypotheses have been proposed: the engagement of the TCR causes a conformational change in the intracellular portion of CD3, which is detected by the intracellular signalling proteins, and/or TCR engagement causes a clustering of CD3, such that the signalling proteins are recruited to the cluster.

Kappler and colleagues generated mice that expressed a mutant form of $C D 3 \varepsilon$, one of the chains that constitute the CD3 molecule, in which the cysteines were replaced with serines in the highly conserved region of the extracellular stalk. The authors found that despite similar levels of TCR expression on CD3E mutant thymocytes compared with those in wild-type mice, there was poor progression from the double negative 3 (DN3) to DN4 stages of thymocyte development, suggesting a defect in pre-TCR signalling ability. Nevertheless, some mature T cells could develop in the thymus, and these populated the periphery through homeostatic proliferation. To determine the TCR signalling capacity of the CD $3 \varepsilon$ mutant

$\mathrm{T}$ cells, the authors measured the levels of extracellular signal-regulated kinase
(ERK) phosphorylation and CD69 expression. After stimulation of the TCR with an antibody, fewer CD $3 \varepsilon$ mutant T cells showed ERK phosphorylation and CD69 upregulation than the wild-type $\mathrm{T}$ cells, suggesting the mutant CD3 could not signal as efficiently as the wild-type CD3.

To determine whether the defective signalling was due to an inability of the mutant $\mathrm{CD} 3 \varepsilon$ to associate with the other $\mathrm{CD} 3$ chains, the authors used a CD3-specific cross-linking antibody to cluster the CD3 molecules. Regardless of CD3 cluster formation, phosphorylation of ERK was lower in $\mathrm{CD} 3 \varepsilon$ mutant mice than wild-type mice, suggesting that instead of a simple clustering mechanism, induction of a conformational change in the intracellular portion of CD3 might constitute part of the CD3-mediated activation signal. How such a signal might be transmitted from the extracellular to the intracellular portion of the protein is still unknown.

Gemma Ryan

ORIGINAL RESEARCH PAPER Wang, Y. et al. A conserved CXXC motif in $C D 3 \varepsilon$ is critical

for T cell development and TCR signaling. PLoS Biol. 7, e1000253 (2009) 